\title{
Techniques for Evaluation of LAMP Amplicons and their Applications in Molecular Biology
}

\author{
Mohammad javad Dehghan Esmatabadi ${ }^{1 \star}$, Ali Bozorgmehr ${ }^{2 \&}$, Hesam Motaleb \\ zadeh $^{3}$, Narges Bodaghabadi ${ }^{1}$, Baharak Farhangi ${ }^{4}$, Sadegh Babashah ${ }^{1 * *}$, Majid \\ Sadeghizadeh ${ }^{1 *}$
}

\begin{abstract}
Loop-mediated isothermal amplification (LAMP) developed by Notomi et al. (2000) has made it possible to amplify DNA with high specificity, efficiency and rapidity under isothermal conditions. The ultimate products of LAMP are stem-loop structures with several inverted repeats of the target sequence and cauliflower-like patterns with multiple loops shaped by annealing between every other inverted repeats of the amplified target in the similar strand. Because the amplification process in LAMP is achieved by using four to six distinct primers, it is expected to amplify the target region with high selectivity. However, evaluation of reaction accuracy or quantitative inspection make it necessary to append other procedures to scrutinize the amplified products. Hitherto, various techniques such as turbidity assessment in the reaction vessel, post-reaction agarose gel electrophoresis, use of intercalating fluorescent dyes, real-time turbidimetry, addition of cationic polymers to the reaction mixture, polyacrylamide gel-based microchambers, lateral flow dipsticks, fluorescence resonance energy transfer (FRET), enzyme-linked immunosorbent assays and nanoparticle-based colorimetric tests have been utilized for this purpose. In this paper, we reviewed the best-known techniques for evaluation of LAMP amplicons and their applications in molecular biology beside their advantages and deficiencies. Regarding the properties of each technique, the development of innovative prompt, cost-effective and precise molecular detection methods for application in the broad field of cancer research may be feasible.
\end{abstract}

Keywords: LAMP - isothermal conditions, evaluation of reaction accuracy - quantitative inspections, various techniques

Asian Pac J Cancer Prev, 16 (17), 7409-7414

\section{Introduction}

Several DNA isothermal amplification methods have been developed in the two past decades. Among them, loop-mediated isothermal amplification technique (LAMP) with its outstanding features has become a noteworthy procedure for specific amplification of a DNA target sequence in molecular biology (Mori and Notomi, 2009). The basic mechanism behind this method relies on Bst DNA polymerase enzyme with its high autocycling strand displacement action. Typically, 4 different primers are used to identify 6 distinct regions on the target gene, which adds highly to the specificity. Briefly, in the initiation of reaction, all four primers, including two inner primers (forward inner primer (FIP) and backward inner primer (BIP)) and two outer primers (F1 and B1) are used, but later, in the extension step, only the inner primers are applied for synthesis of new DNA strands. The stem-loop DNA structures with several inverted repeats of the target and cauliflower-like structures with different lengths are the final products of reaction.
The most common access method for evaluation of amplification reaction is to naked-eye inspection of turbidity derived from precipitation of magnesium ions in the environment during reaction (Mori et al., 2001). Although using of two or three pairs of distinct primers complementary to the four or six discrete regions of the target sequence decrease the possibility of non-relevant amplification, to the benefit of more accurate analysis of amplified products or quantitative measurements of amplification reaction, it is inevitable to append other analytical procedures to amplification process.

Here, we describe the most widely-known methods (such as post-reaction agarose gel electrophoresis, using intercalating fluorescent dyes, real-time turbidimetry, addition of cationic polymers to the reaction mixture, polyacrylamide gel-based microchamber, lateral flow dipstick, fluorescence resonance energy transfer (FRET), enzyme-linked immunosorbent assays and nanoparticlebased colorimetric tests) in addition to their applications, privileges and deficiencies. These techniques vary from each other in their requirements for sample volume,

${ }^{1}$ Department of Molecular Genetics, Faculty of Biological Sciences, Tarbiat Modares University, Tehran, ${ }^{2}$ Department of Neuroscience, Iran University of Medical Science, ${ }^{3}$ Department of Genetics, Faculty of Basic Sciences, Science and Research Branch, Islamic Azad University, Tehran, ${ }^{4}$ Department of Genetics, Faculty of Biology, University of Guilan, Rasht, Iran ${ }^{\star}$ Equal *Contributors **For correspondence:babashah@modares.ac.ir,sadeghma@modares.ac.ir 
specimen preparation, disposable reagents and strategies of detection. It is clear that there is probably no one example that is best convenient or suited for all possible situations.

\section{Methodological Approaches}

\section{Turbidity evaluation after amplification reaction}

It was surveyed that when DNA is amplified by the LAMP reaction, the turbidity derived from precipitation of is produced during progress of the reaction. Chemical and spectroscopic analyses indicated the produced precipitate as magnesium pyrophosphate.

Production of precipitate in the LAMP reaction can be described by the following reactions:

a) $(\text { DNA })_{n-1}+$ dNTP $\rightarrow(\text { DNA })_{n}+\mathrm{P}_{2} \mathrm{O}_{7} 4-$

b) $\mathrm{P}_{2} \mathrm{O}_{7} 4-+2 \mathrm{Mg}^{2+} \rightarrow \mathrm{Mg}_{2} \mathrm{P}_{2} \mathrm{O}_{7}$

In amplification process, pyrophosphate ions are released from dNTPs as a byproduct (a). Pyrophosphate ions react with magnesium ions in the LAMP reaction solution producing a precipitate (b).

When a reaction mixture containing amplified DNA centrifuged, precipitate cumulate at the bottom of the vessel and can easily be corroborated with naked eyes. Also, sometimes detection of the LAMP reaction can be accomplished by judging the presence of cumulated precipitate instantly after reaction without centrifugation. In this approach, there is no need to apply any manifestation reagent such as fluorescent intercalating dyes, which are often utilized in DNA detection but environmentally disagreeable and highly pernicious. In addition, a simple and economically feasible DNA detection device could be set up (Mori et al., 2001). However, amplification of a non-relevant sequence is not distinguishable and more ever, factual quantitative measurements are not applicable by this strategy and sometimes, the signal in this detection system is rather weak for visual detection.

\section{Post-reaction agarose gel electrophoresis}

Analysis of amplified DNA is usually carried out by agarose gel electrophoresis, followed by fluorescent staining and inspection under UV light. Due to the formation of stem-loop shapes of varying stem length and cauliflower-like structures with multiple loops formed by sequentially inverted repeats of the target sequence, the LAMP amplicon of the positive sample displays many bands of different sizes upon agarose gel electrophoresis (Notomi et al., 2000). So, amplified DNA produce a ladder-like pattern on the gel and this differentiates between LAMP products and the amplicons of other amplification methods. This technique is common and available in almost all molecular laboratories, but inability to precise detection and quantification of amplified DNA and also high risk of contamination of negative samples by amplicons are the most outstanding defects beside it.

\section{Using of fluorescent intercalating dyes}

For better visual evaluation of the reaction outcome, DNA intercalating dyes such as SYBR green, Picogreen or propidium iodide could be added to the reaction solution after the amplification is completed. When the LAMP process is positive, a color change is observable (Table 1). For example in 2005, Parida et al. (2005) applied the SYBR Green dye for inspection of a real time RTLAMP product to diagnosis of Dengue Virus Serotypes (Parida et al., 2005). This strategy is affordable, rapid and quantitative and could be evaluate without high-tech instruments. Nevertheless, the colorimetric assay using the intercalating dye is associated with an elevated risk of contamination of other following LAMP reaction solutions since it necessitates opening of the tubes. On the other hand, the described dyes are environmentally inadvisable.

\section{Real-time turbidimetery}

In 2004, Mori et al. designed an apparatus for a realtime turbidimetery method, capable of monitoring the LAMP reaction mixtures and measuring the turbidity production of multiple samples simultaneously without interruption (Mori et al., 2004). So, the method could quantify amplified DNA without need for any detection reagents and this led to develop assays for quantitative analysis of DNA. However, the outcomes were achievable in the laboratory setting, and it is debatable whether these results also applicable to actual specimens. For example, in detection of pathogens present in the blood or intestinal secretions it is necessary to determine the limit to which any restrictive agents present in the samples or sampling preciseness has an effect on quantification process. On the other hand, although the developed method has shown considerable advantages, need for a turbidimeter apparatus and inability to determination of amplification reliability,

Table 1. DNA binding Dyes and their Interpretation

\begin{tabular}{lcc}
\hline Agent & Positive & Negative \\
\hline SYBR green & Green & Orange \\
Propidium iodide & Light Pink & Red \\
Picogreen & Green & Orange \\
\hline
\end{tabular}

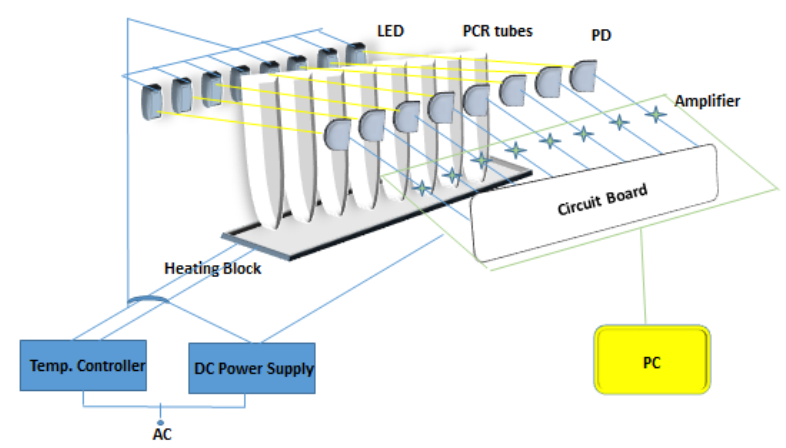

Figure 1. Block Diagram of the Turbidimeter. Eight PCR tubes can be fixed between eight LED and PD. The tubes are heated to the optimum temperature for LAMP reactions (60$65 \pm 0.5^{\circ} \mathrm{C}$ ) by a heating block that is designed to avoid blocking the light path. Each light $(650 \mathrm{~nm} ; 2 \mathrm{~mm}$ I.D. $)$ emitted by eight light emitting diodes (LED) passes through the PCR tube and illuminates the photodiodes (PD) opposed to the LED. The mean optical path length is about $2.5 \mathrm{~mm}$. AC, alternating current; DC, direct current; LED, light emitting diode; PD, photo diode; PC, personal computer 
impede its usage.

The turbidimeter apparatus invented by Mori et al. is shown in Figure 1.

\section{Cationic polymers}

In 2006, an innovative approach for the sequencespecific visual identification of very low quantities of nucleic acids was developed based on addition of cationic polymers to LAMP amplified products and inspection of

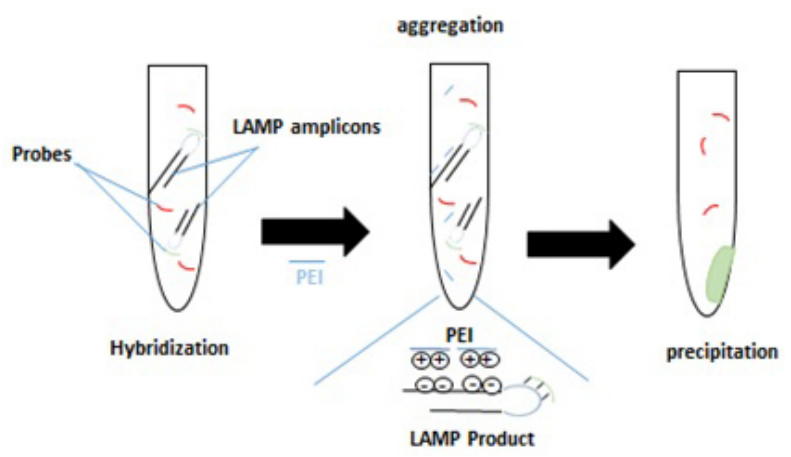

Figure 2. Precipitation Titration of LAMP Amplicons by Adding PEI. When a LAMP reaction in presence of an appropriate fluorescent-labeled probe progresses, the probe will hybridize to the complementary loop region of produced amplicon. By adding optimized amount of PEI to the reaction vessel after amplification, the positive charge of PEI neutralizes the negative charge of the amplified DNA and constitute to an insoluble LAMP product-PEI complex formation. Finally, the pellet is irradiated with excitation light and the labeled fluorescent probe hybridized to LAMP products produces fluorescence. Red probe, unattached; Gray probe, attached

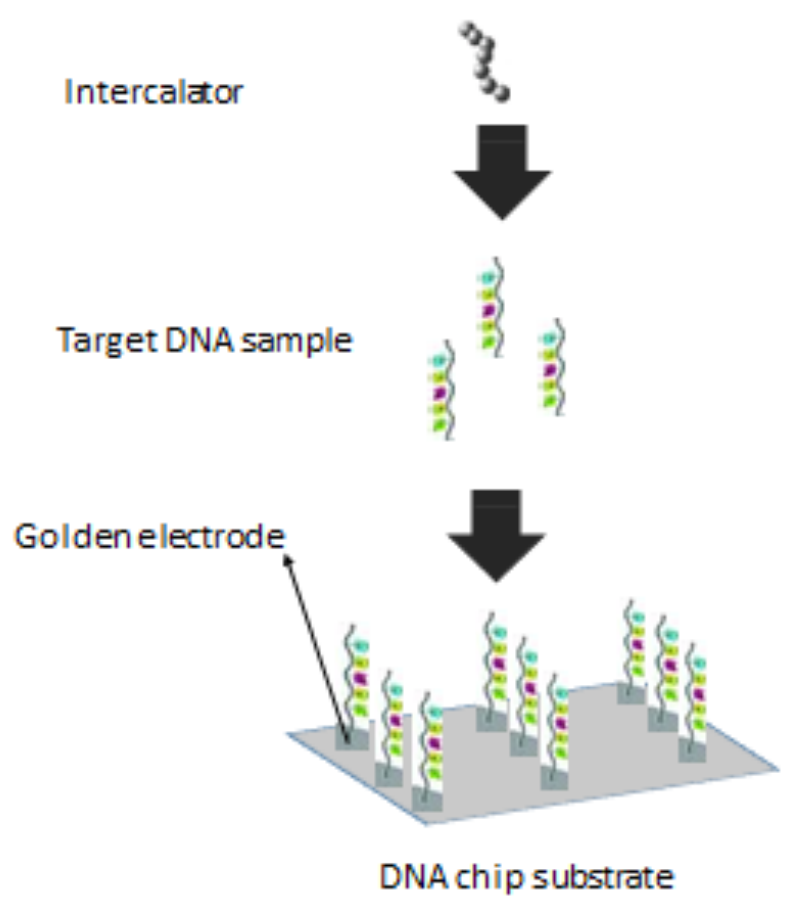

Figure 3. Detection principle of the electrochemical DNA chip. DNA probes with known Sequences are Immobilized on Golden Electrodes. Target DNA hybridizes only to probe DNA with complementary sequence. Intercalator (For example Hoechst 33258) binds only to doublestrands DNAs. Electrical current detected by applying voltage to electrodes precipitation reaction (Mori et al., 2006). The DNA target template was amplified by the LAMP reaction under the existence of the fluorescent dye-labeled oligonucleotide probe. At the end of the LAMP process, appropriate amount of the low molecular weight polyethylenimine (PEI) was incorporated to the reaction environment, led to the precipitation of the insoluble LAMP ampliconPEI complex. The precipitate emitted fluorescence proportionate by the amplified nucleic acid targets and it could be detected on a conventional UV illuminator (Figure 2). However, development of a technique for adding PEI in a closed system is needed to prevent carryout contamination and put this method to practical use.

\section{Electrochemical chip}

A DNA chip is an apparatus in which large amounts of DNA probes are situated on glass or silicon. A technique using an electrochemically active intercalator, Hoechst 33258 and DNA probe immobilized on a gold electrode was developed for simultaneous genotyping of SNPs by combining the LAMP method and the electrochemical DNA chip by Nakamura et al. (2007). This method requires no labeling step and expensive signal transduction equipment. Oligonucleotide probes with a thiol group at the 3' ends were obtained and each working electrode was spotted with probe solution. The DNA chip was covered with a reaction chamber. The LAMP products were reacted with the DNA probes on the electrodes and the hybridization reaction was carried out. Subsequently, the chip was reacted with Hoechst 33258 and then, the anodic peak current derived from Hoechst 33258 was measured by a voltammeter (Figure 3 ). This method is advantageous for realizing an automated system, because the reaction part and the detection part are unified and simple.

\section{polyacrylamide gel-based microchamber}

Lam et al. (2008) developed a novel fluorescent imaging-based method for scrutiny the LAMP reaction

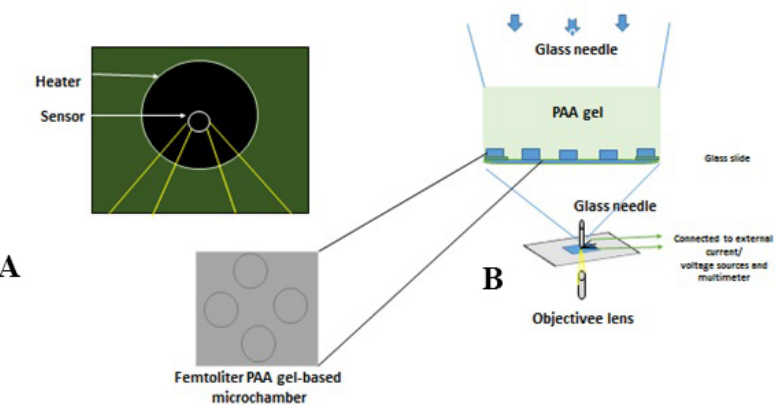

Figure 4. A) Microheater pattern: The microheater system comprised of circular heater and a temperature sensor B) Experimental setup with microscopic view of PAA gel-based microchamber: sample solution was initially deposited on a glass slide, printed with the microheater. The PAA gel-based microchamber was then placed above the sample solution and a glass needle was lowered to press the upper surface of the gel against the glass slide. This way, only one or two DNA templates would be trapped within a single microchamber. Finally, SYBR green II dye was applied to cover the external of the PAA gel, keeping it hydrated in order to retain its structure and encapsulation capability. The microchambers located within the circumferential region bounded by the heater pattern were observed 


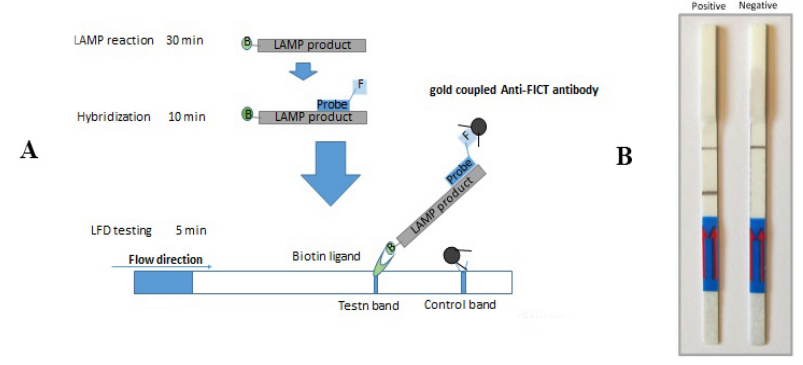

Figure 5. Lateral flow Dipstick Las-LAMP Evaluation. (A) Lateral Flow Dipstick Las-LAMP procedure: LAMP reaction is performed using a biotinilated FIP primer. After 30 minutes of initial incubation at $65^{\circ} \mathrm{C}$, a specific FITC-labelled probe is added to the reaction mixture and incubated for another 10 minutes at the same temperature. This step produces a dual labeled LAMP product. Finally, detection buffer containing Rabbit Anti-FITC antibodies coupled with colloidal gold is mixed with the reaction mixture, and the LFD strip is inserted into the tube. In a positive reaction, double labeled LAMP products migrates with the buffer flow and are retained at the Test Band by a biotin ligand. The gold coupled Anti-FICT antibody binds to the FITC molecule at the probe and a dark red band develops over the time. In the case of a negative reaction no products are generated and such process does not have place. An Anti-Rabbit antibody at the Control Band retains some of the unbound gold-conjugated antibody and produces a Control Band that should be always visible. (B) Evaluation of results using the Lateral Flow Dipstick device

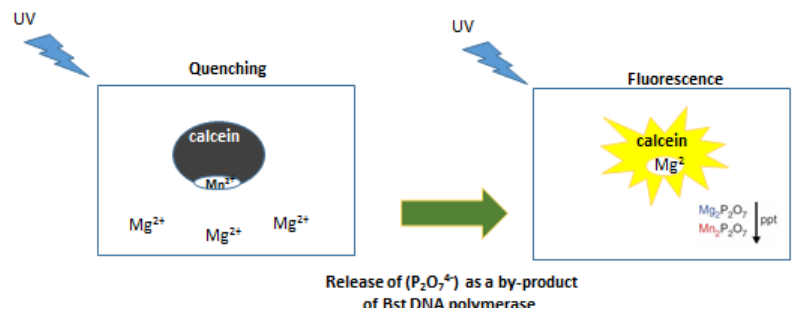

Figure 6. Principle of Detection Using Calcein. In the DNA amplification reaction by Bst DNA polymerase, pyrophosphate ions are released as a by-product from dNTPs. The calcein in the reaction mixture initially combines with manganous ion (Mn2+) and remains quenched. By preceding the reaction, manganous ion is deprived of calcein by the produced pyrophosphate ions (P2O74-), which lead to fluorescence emission. Consequently, the free calcein is apt to combine with magnesium ion $(\mathrm{Mg} 2+)$ in the reaction solution and it corroborates the fluorescence emission

at a single molecule level in 2008 (Lam et al., 2008). The procedure was directed in a polyacrylamide gel based microchamber where a sole DNA template, freely suspended in a mixture including specific primers and DNA polymerase (Figure 4). With the purpose of switch on the amplification reaction, a microheater was applied for localized heating and this strategy established the effective taking advantage of negligible amount of templates and primers, and the on-average decrease in the whole detection time to about $50 \mathrm{~min}$ in a one-step operation. Also, possibility of direct observation of reaction progression using optical microscopy potentiates this method as a simple substitution to conventional procedures for genetic analysis and diagnosis in the clinical laboratories. However, setting up the apparatus might be the difficult step of this approach.

Lateral flow dipsticks (LFD)

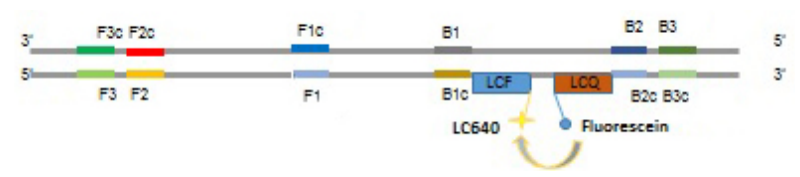

Figure 7. Location and Sequence of LAMP Primers and FRET Hybridization Probes. Two additional oligonucleotide probes was designed complementary to one loop region of LAMP amplicon and added to the LAMP reaction mixture. When both probes hybridize simultaneously to the target sequence, the transmission of fluorescence energy from the fluorescein dye to the LC640 acceptor dye, causes a change in the emission wavelength of the emission of the LC640 dye in the reaction mixture

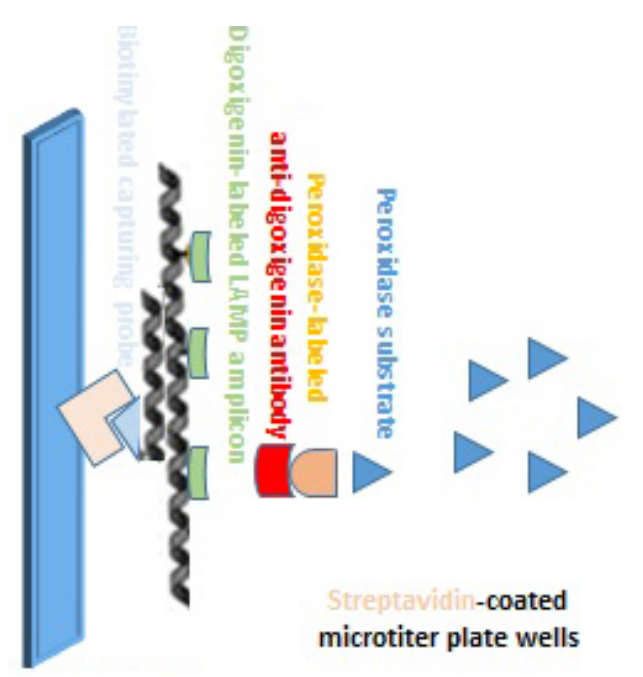

Figure 8. The Main Strategy used in the Development of the LAMP/ ELISA Technique. Biotinylated capturing probes help to fix LAMP amplicons to streptavidin-coated microtiter plate wells. Peroxidase-labeled anti-digoxigenin antibody interacts with DIG-11-dUTPs in the apmlicon. Upon addition of substrate, in the positive sample, the color changes from yellow to blue

Loop-mediated isothermal amplification integrated with chromatographic lateral flow dipsticks (LFD) by Nimitphak in 2008 and provided a simple detection procedure for detection of PmDNV-infected shrimp within 75 min (Nimitphak et al., 2008). In this approach, Biotinylated LAMP products achieve and then hybridize isothermally in amplification reaction temperature, with an FITC-labeled oligonucleotide probe that is complementary to the outside the primer regions. The biotinylated, FITC-labeled LAMP amplicons attach with gold-labeled, anti-FITC and the triple-labeled complex captures by fixed biotin-binding proteins to yield a red nano-gold stripe at the LFD test (Figure 5). The reports shown that integration of LAMP and LFD methods requires relatively inexpensive equipment, is highly sensitive and using a detection probe makes it very specific. Furthermore, The LFD does not require high-tech instrumentation for the reason that the user directly dips the LFD into an appropriately buffered LAMP medium.

\section{Using calcein as magnesium ion indicator}

Calcein could be added to a LAMP reaction solution to inspection of reaction progress. However, as it was resulted, the reduction of magnesium ions induced by the 


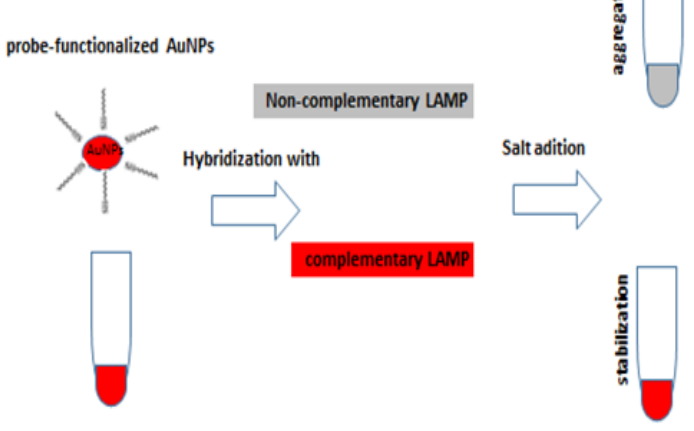

Figure 9. Schematic Illustration for the Detection Method based on Probe-Functionalized AuNPs. The DNA-targeted specific probes are functionalized to AuNPs through the disulfide interactions. The probe-functionalized AuNPs are hybridized with the DNA target (Complementary and non-complementary LAMP product). The addition of salt to change the solution color. A sample is recorded as a negative if the color of solution changes to blue/gray, and vice versa

LAMP reaction was not sufficient to change the color to a naked-eye detectable level, therefore the amplification reaction must be monitored by the colorimetric methods and adding manganous ion to the reaction solution, which forms an insoluble salt with the pyrophosphate generated as a reaction by-product, but quenches the reaction by complexation with calcein. At first, the calcein combines with ion $\mathrm{Mn}^{2+}$ and remains quenched. By proceed the amplification; manganous ion is dispossessing from calcein by the generated pyrophosphate ions, which constitute the radiation of fluorescence signals and the free calcein is able to forming complex with magnesium ions in the reaction mixture, so that it corroborates the fluorescence emission (Tomita et al., 2008) The principles of the process is shown in Figure 6. The ability to realtime detection of amplified product that reduces the cross-contamination risk is the most prominent advantage of this approach. However, this technique is instrumentdependent for evaluation.

\section{Real-time colorimetric detection by using hydroxy naphthol blue (HNB)}

In 2009, Goto et al reported a real-time colorimetric assay for assessment of LAMP reaction progression using HNB (hydroxy naphthol Blue) as an indicator for the $\mathrm{Mg}^{2+}$ ion concentrations (Goto et al., 2009). Since $\mathrm{Mg}^{2+}$ ion concentration decreases as the LAMP reaction progresses, the LAMP reaction can be quantified by measuring the $\mathrm{Mg}^{2+}$ ion concentration in the reaction solution. The color of HNB changes from violet (negative reaction) to sky blue (positive reaction) due to the chelation of $\mathrm{Mg}^{2+}$ ions by dNTPs. Opening the reaction tube not required in this approach and this reduces the risk of cross-contamination. Easy operation, no need for special equipment, superior sensitivity and speed and suitability for high-throughput DNA and RNA detection are the other prominent advantages of this strategy, but the reliability of the specific target sequence amplification is not detectable.

\section{Fluorescent resonance energy transfer (FRET)}

The fluorescent resonance energy transfer (FRET) probe technology was applied to detection of LAMP products by Pin-Hsing Choua et al in 2011 as the FRETLAMP assay for diagnosis of white spot syndrome virus (WSSV) of penaeid shrimp (Chou et al., 2011). In that study, two specific FRET probes designed to aim at one loop region within LAMP amplicons and were added to the same LAMP reaction mixture. The FRET-based probes as may reasonably be presumed hybridize to the complementary single-stranded loop region of LAMP products constitute real-time transmission of the FRET signal. For real-time detection of LAMP amplicons, the 24-nt FRET "acceptor" probe (LCF) was labeled with LightCycler Red 640 at the 5' end and modified by phosphorylation at the 3 ' end to avoid undesired primer extension. The 3'-fluorescein-labeled "donor" probe (LCQ) is 21-nt long and binds at 2 bases 5' to the LC640-labeled "acceptor" probe. When both probes hybridize to the target sequence, the fluorescence energy is transferred from the fluorescein dye to the LC640 acceptor dye, leading to the emission of the LC640 dye (Figure 7). The established assay could be easily set up by an inexperienced person and fulfilled in a short period of time (50-80 min). In addition, this offers good points in both target amplicon-specific detection and a low probability risk of getting false positive results. Furthermore, the notation of minor variations in kinetics and intensity of the FRET signals in repeated experiments demonstrated that the FRET-LAMP reaction is relatively reproducible. However, monitoring the FRET hybridization probes signals was dependent on a custom-built real-time thermocycler though LAMP is ranked as an isothermal amplification reaction.

\section{Enzyme-linked immunosorbent assay (ELISA)}

Loop-mediated isothermal amplification in conjunction with enzyme-linked immunosorbent assay (LAMPELISA) developed in 2012 to provide a sensitive, specific and convenient method as diagnostic kit for detection of Salmonella serogroup D strains (Ravan and Yazdanparast, 2012). The LAMP-ELISA is based on direct incorporation of labeled nucleotides, Digoxigenin-11-dUTP in amplified products during the LAMP-amplification process following by their hybridization to specific capturing oligonucleotide probes, and ultimately, detection of the captured amplicons by immunoassay assay (Figure 8). Regarding the assay time, the LAMP-ELISA could be fulfilled in a few hours with the capability to detect simultaneously up to several hundred samples. In 2013, The LAMP-ELISA method was improved by using a pair of Biotinylated loop primers and direct hybridization of amplicons to the streptavidin-coated ELISA plates (Ravan and Yazdanparast, 2013). This strategy reduces the overall time of detection and risk of cross-contamination.

\section{DNA-functionalized gold nanoparticle}

The combination of loop-mediated isothermal amplification (LAMP) and probe-functionalized gold nanoparticles (AuNPs) was developed and applied for the detection of white spot syndrome virus (WSSV) by Seetang-Nun et al. (2013). The basic strategy of this method depends on the stability characteristics 
of the probe-functionalized gold nanoparticles upon hybridization with their complementary target DNA opposed to aggregation following increasing the environmental salt concentration. If the DNA sample is not complementary with the detection probe, the probefunctionalized AuNPs will be aggregated and proceeding by the change of solution color from red to blue/gray and shift of the surface plasmon peak to longer wavelength, while in the positive sample, no considerable change is occurred Figure 9. Simplicity, swiftness and capability to quantitative evaluation of target DNA, are the most effective advantages of this assay. But like many other detection techniques, the risk of cross-contamination and getting false positive results is high in this approach.

\section{Conclusions}

Loop-mediated isothermal amplification (LAMP) was developed as an isothermal amplification method with the ability of detecting only a few copies of target nucleic acid by self-recurring strand-displacement DNA synthesis. The detection and evaluation of LAMP products has been initially achieved with several simple techniques such as agarose gel electrophoresis stained with ethidium bromide (Notomi et al., 2000), inspecting of the turbidity derived from precipitation of magnesium pyrophosphate as a reaction by-product (Mori et al., 2001), and by incorporation of DNA intercalating agents such as SYBR Green I following by measuring the fluorescence (Soliman and El-Matbouli, 2006). Over the last few years, several more accurate techniques like lateral flow dipstick (LFD), enzyme-linked immunosorbent assay (ELISA) and non-cross-linking assay are developed and applied in this field. However each method has its deficits beside its advantages. Detachment of detection process from amplification reaction can lead to increase cross-contamination risk and the probability of getting false-positive results. On the other hand, direct detection of amplified DNA without opening the amplification reaction vessel is a complex and frail procedure.

In this article, we reviewed the best-known techniques for LAMP amplified products detection and evaluation. The presented information can be useful for researchers who are trying LAMP as a diagnostic tool especially in the fields of microbiology, virology, mycology and biotechnology. Regarding the properties of each technique and with the other amplification methods attributes in mind, the design and development of new integrated assays may be possible in the future.

\section{Acknowledgement}

The authors report no conflicts of interest

\section{References}

Chou PH, Lin YC, Teng PH, et al (2011). Real-time targetspecific detection of loop-mediated isothermal amplification for white spot syndrome virus using fluorescence energy transfer-based probes. J Virol Methods, 173, 67-74.

Goto M, Honda E, Ogura A, et al (2009). Colorimetric detection of loop-mediated isothermal amplification reaction by using hydroxy naphthol blue. Biotechniques, 46, 167-72.

Lam L, Sakakihara S, Ishizuka K, et al (2008). Loop-mediated isothermal amplification of a single DNA molecule in polyacrylamide gel-based microchamber. Biomed Microdevices, 10, 539-46.

Mori Y, Hirano T, Notomi T (2006). Sequence specific visual detection of LAMP reactions by addition of cationic polymers. BMC Biotechnol, 6,3 .

Mori Y, Kitao M, Tomita N, et al (2004). Real-time turbidimetry of LAMP reaction for quantifying template DNA.J Biochem Biophys Methods, 59, 145-57.

Mori Y, Nagamine K, Tomita N, et al (2001). Detection of loopmediated isothermal amplification reaction by turbidity derived from magnesium pyrophosphate formation. Biochem Biophys Res Commun, 289, 150-4.

Mori Y,Notomi T(2009).Loop-mediated isothermal amplification (LAMP): a rapid, accurate, and cost-effective diagnostic method for infectious diseases. J Infect Chemother, 15, 62-9.

Nakamura N, Ito K, Takahashi M, et al (2007). Detection of six single-nucleotide polymorphisms associated with rheumatoid arthritis by a loop-mediated isothermal amplification method and an electrochemical DNA chip. Anal Chem, 79, 9484-93.

Nimitphak T, Kiatpathomchai W, Flegel TW (2008). Shrimp hepatopancreatic parvovirus detection by combining loop-mediated isothermal amplification with a lateral flow dipstick. J Virol Methods, 154, 56-60.

Notomi T, Okayama H, Masubuchi H, et al (2000). Loopmediated isothermal amplification of DNA. Nucleic Acids Res, 28, 63.

Parida M, Horioke K, Ishida H, et al (2005). Rapid detection and differentiation of dengue virus serotypes by a real-time reverse transcription-loop-mediated isothermal amplification assay. J Clin Microbiol, 43, 2895-903.

Ravan H, Yazdanparast R (2012). Development and evaluation of a loop-mediated isothermal amplification method in conjunction with an enzyme-linked immunosorbent assay for specific detection of Salmonella serogroup D. Anal Chim Acta, 733, 64-70.

Ravan H, Yazdanparast R (2013). Loop region-specific oligonucleotide probes for loop-mediated isothermal amplification-enzyme-linked immunosorbent assay truly minimize the instrument needed for detection process. Anal Biochem, 439, 102-8.

Seetang-Nun Y, Jaroenram W, Sriurairatana S, et al (2013). Visual detection of white spot syndrome virus using DNAfunctionalized gold nanoparticles as probes combined with loop-mediated isothermal amplification. Mol Cell Probes, 27, 71-9.

Soliman H, El-Matbouli M (2006). Reverse transcription loopmediated isothermal amplification (RT-LAMP) for rapid detection of viral hemorrhagic septicaemia virus (VHS). Vet Microbiol, 114, 205-13.

Tomita N, Mori Y, Kanda H, et al (2008). Loop-mediated isothermal amplification (LAMP) of gene sequences and simple visual detection of products. Nat Protoc, 3, 877-82. 